\title{
Nachahmenswerte Teamarbeit
}

\author{
MOBILE GERIATRISCHE REHABILITATION IN KARLSRUHE Zwei multiprofessionelle \\ Teams touren Tag für Tag für ihre Patienten durch Karlsruhe. Dabei bieten sie weit \\ mehr als übliche Hausbesuche. Sie arbeiten intensiv und erfolgreich mit den Angehö- \\ rigen und als Kollegen zusammen. ergopraxis schaute dem außergewöhnlichen \\ Team einen Tag lang über die Schulter.
}

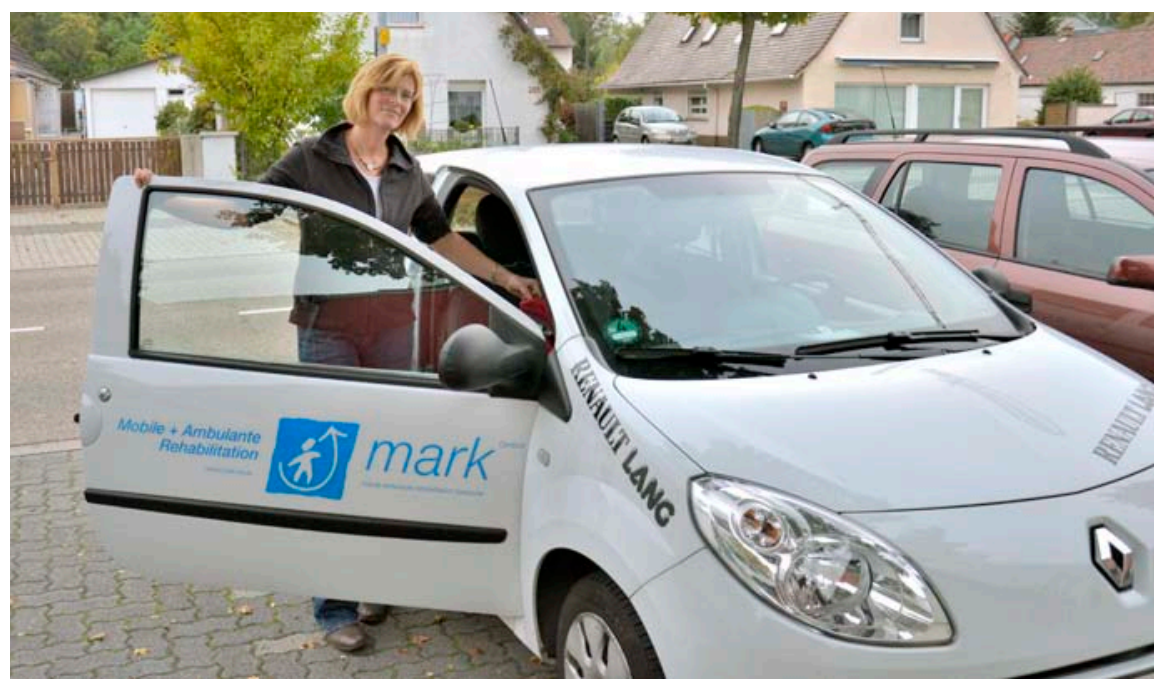

Ergotherapeutin Silvia Hark auf dem Weg zu ihren Patienten

\section{D}

ienstagnachmittag, 14 Uhr. Ich darf bei der wöchentlichen Teamsitzung im Karlsruher Hardtwaldzentrum dabei sein. Dort sitze ich mit Christian Aldus, dem Ärztlichen Leiter der Mobilen Geriatrischen Rehabilitation Karlsruhe, einer Ergo-, einer Physiotherapeutin, der Logopädin und der Sozialarbeiterin im Besprechungsraum. Jeder hat seinen Stapel Patientenakten vor sich. In der Dienstagsrunde geht das Team jeden einzelnen Patienten durch. Dafür haben sie eine Stunde Zeit. Ein straffes Programm. Christian Aldus gibt den Anstoß: „Beginnen wir mit Herrn Adler. Seiner Frau war ja wichtig, dass der Transfer leichter geht. Welche Fortschritte gibt es dazu?" Daraufhin fragt die Physiotherapeutin an die Ergotherapeutin gewandt: „Wenn Herr Adler* im Bett liegt, weiß ich, wie ich vorgehen muss, aber wie machst du das auf dem Sofa mit ihm?“ Die Ergotherapeutin erklärt ihrer Kollegin, worauf sie beim Transfer achtet, und schlägt vor: „Üb doch du mit ihm im Bett, das Sofa übernehme ich." Dann schaltet sich die Logopädin ein: „Mir sagte Herr Adler, er habe früher einen guten Wortschatz gehabt und wolle Fremdwörter üben. Das und Hirnleistungstraining habe ich mit ihm gemacht, aber eine Verlängerung braucht er in der Hinsicht nicht.“ Daraufhin entgegnet die Ergotherapeutin: „In meinem Bereich gibt es noch Ziele, die wir im Rahmen einer Verlängerung erreichen werden. Diese würden auch eine Erleichterung für die Ehefrau schaffen. Da könnte ich ja dann deine Inhalte einfließen lassen.“ Als Letztes berichtet auch die Sozialarbeiterin: „Ich habe die Eheleute über einige rechtliche Dinge informiert, unter anderem über Patientenverfügungen. Die Unterlagen habe ich zusammengestellt und würde sie dir gerne morgen für die beiden mitgeben.“
Fasziniert verfolge ich die Teamsitzung. So habe ich mir interdisziplinäre Zusammenarbeit immer vorgestellt. Hier läuft die Therapie tatsächlich Hand in Hand. Keiner macht sich seinen Arbeitsbereich streitig, alle ziehen an einem Strang, und keiner fühlt sich auf den Schlips getreten, wenn Zuständigkeitsbereiche überschritten werden. Die Kommunikation läuft auf Augenhöhe, auch mit Christian Aldus.

Das Angebot ist einzigartig in BadenWürttemberg: An zwei Karlsruher Standorten, im Norden und im Süden, betreuen zwei multiprofessionelle Teams derzeit fünfzehn Patienten. Die Ergotherapeuten, Physiotherapeuten, Logopäden, Pflegetherapeuten, Sozialarbeiter, Neuropsychologen und Ernährungsberater kommen dann zum Einsatz, wenn jemand nur im häuslichen Bereich behandelt werden kann.

Reha in der gewohnten Umgebung > Das Durchschnittsalter der Patienten liegt bei 80 Jahren. Sie kommen entweder nach einem akuten Ereignis wie einem Schenkelhalsbruch aus der Klinik oder über den Hausarzt zur Mobilen Geriatrischen Reha. Besonders ist bei ihnen, dass sie durch die Behandlung zu Hause bessere Chancen auf eine erfolgreiche Rehabilitation haben als in einer stationären Einrichtung. Häufig sind das Menschen mit einer Demenz, mit starken Sehbehinderungen oder Patienten mit psychischen Störungen, die ihr vertrautes Umfeld brauchen. Meist leben sie in gezielt angepassten Lebensverhältnissen. Der Aufenthalt in einer fremden Umgebung mit fremden Bezugspersonen würde nicht zum Rehabilitationssprozess beitragen. In diesen Fällen schließt die mobile geriatrische Reha die Lücke zwi- 


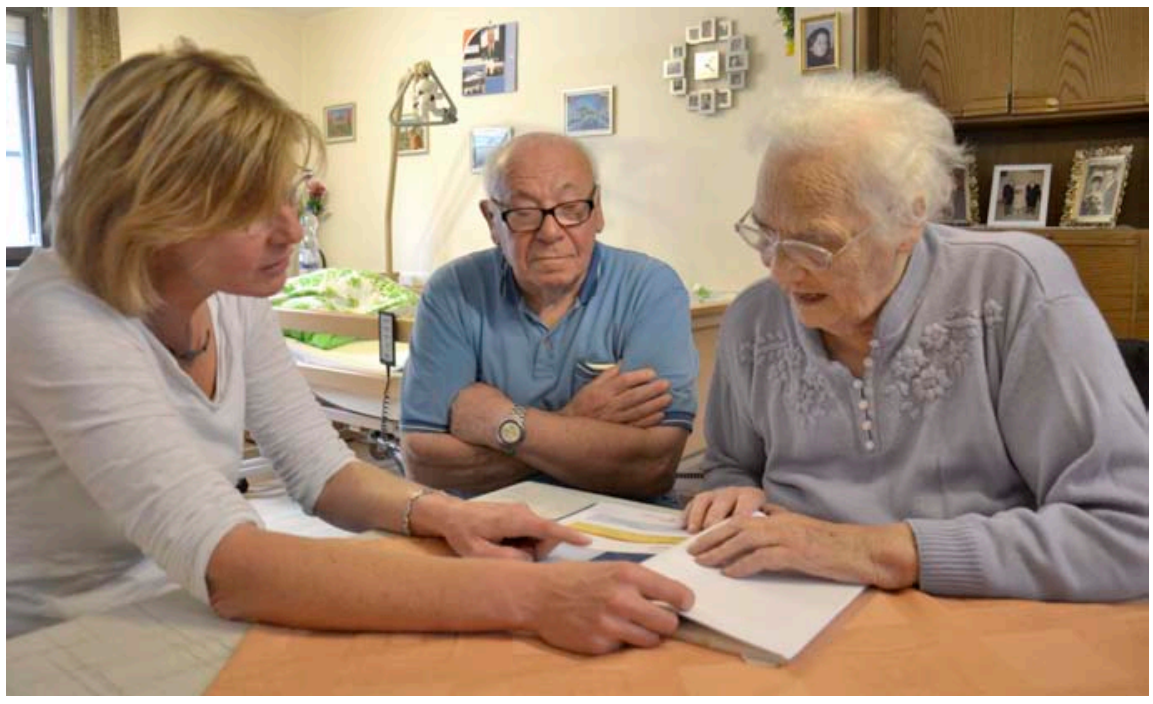

Bei ihren Hausbesuchen berät Silvia Hark unter anderem über Hilfsmittelangebote, um den Alltag der Patienten und ihrer Angehörigen zu erleichtern.

schen einer regulären ambulanten und einer stationären Versorgung und stellt sich auf die Bedürfnisse der Patienten ein.

Das Therapeutenteam ist an die Rahmenverträge mit den Krankenkassen gebunden. Insgesamt stehen ihm pro Patient 40 Behandlungseinheiten über einen Zeitraum von etwa sechs Wochen zur Verfügung. Falls erforderlich, wird die Therapie um weitere Einheiten verlängert.

Zu Beginn einer Rehabilitationsmaßnahme übernimmt Dr. Aldus den ersten Hausbesuch und untersucht die Patienten. Dabei achtet er nicht nur auf deren physische und kognitive Verfassung, sondern verschafft sich auch einen ersten Eindruck von den häuslichen Gegebenheiten und unterhält sich mit den Angehörigen. Dazu führt er das geriatrische Assessment mit Testelementen wie den Mini-MentalStatus-Test, den DemTect oder den ChairRising-Test durch. Daraufhin stellt er den Rehabilitationsplan auf, entscheidet also, welche Therapeuten aus dem interdisziplinären Team beteiligt sein sollen, und leitet ihnen seine ersten Eindrücke und Ergebnisse weiter. So sind diese für den Erstkontakt gerüstet und können mit der Behandlung beginnen.

Zu Hause geht es um Teilhabe $>$ Bei meinem Besuch habe ich die Gelegenheit, mit der Ergotherapeutin Katrin Storbeck aus dem Süd-Team zu sprechen. Sie arbeitet zu 50 Prozent in der Mobilen Geriatrischen Reha und zu 50 Prozent in der Klinik für Rehabilitative Geriatrie. Das heißt, die Unterschiede von der stationären zur ambulanten Rehabilitation erlebt sie tagtäglich. „Zu Hause treten ganz andere Probleme auf, als sie in der stationären Reha für die Patienten absehbar wären“, erklärt sie. Wenn sie stationär untergebrachte Patienten nach ihren Zielen fragt, dann möchten sie häufig „einfach“ wieder gesund werden oder besser laufen können. Patienten, die in ihrem gewohnten Umfeld bleiben, erleben ihre Einschränkungen jedoch direkt im Alltag und können danach ihre Ziele ausrichten. Sie möchten ihre Enkel wieder besuchen, wieder in die Tagespflege gehen, die Treppe vor der Haustüre bewältigen oder sich wieder alleine waschen und anziehen können. Der Arbeitsauftrag im gewohnten Umfeld unterscheidet sich also deutlich von dem in der Klinik. Die Ziele der Patienten richten sich zu Hause stark auf Partizipation und Teilhabe. So haben sie ein klares Ziel vor Augen und können besser nachvollziehen, woran sie in der Therapie später arbeiten. Falls die Patienten zur Zielformulierung kognitiv nicht mehr in der Lage sind, übernehmen das die Angehörigen. Als Basis führt die Ergotherapeutin zu Beginn einer Behandlung das Ergo-Ass durch, meist auch den Uhrzeichentest.

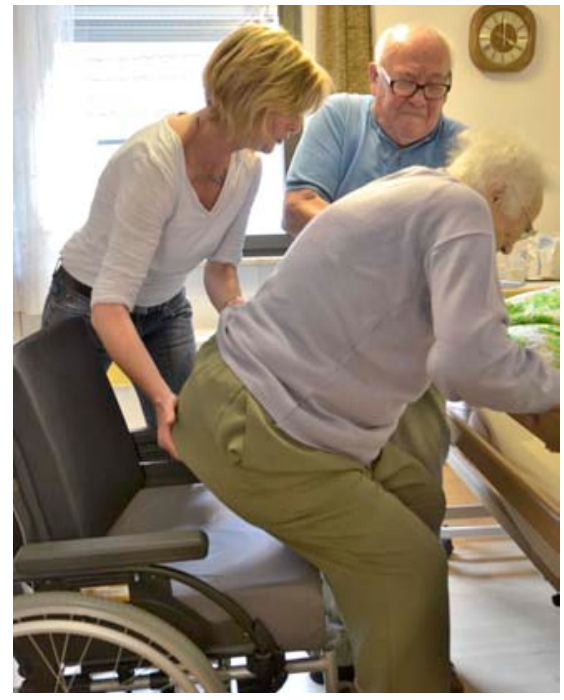

Anleitung spielt eine große Rolle. Nur so kann der Transfer in den Alltag gelingen.

Damit die Behandlungen besser verteilt sind, haben die Therapeuten unterschiedliche Arbeitszeiten. So ist Katrin Storbeck morgens für die Mobile Geriatrische Reha unterwegs und arbeitet nachmittags in der Klinik. In der Zeit sind dann ihre Kollegen auf Hausbesuch. Ein typischer Arbeitstag beginnt für die Ergotherapeutin im Büro. Dort schaut sie, ob ihre Kollegen oder einer ihrer Patienten wichtige Infos oder eine Terminabsage hinterlassen haben. Dann macht sie sich auf den Weg. In der Regel sieht sie ihre Patienten zwei- bis dreimal pro Woche und kann so eine sehr intensive Beziehung zu ihnen aufbauen. Da sie jeweils eine Dreiviertelstunde vor Ort ist, bevor sie zum nächsten Patienten fährt, schafft sie drei Besuche pro Vormittag. Anschließend erledigt sie die Dokumentation, kümmert sich um den Stundenplan für die darauffolgende Woche, leitet Hilfsmittelverordnungen in die Wege, erledigt Telefonate mit Sanitätshäusern, Krankenkassen, Angehörigen und Hausärzten.

Intensive Angehörigenarbeit > Was macht die Mobile Geriatrische Reha so besonders, frage ich Katrin Storbeck. „Die intensive Angehörigenarbeit“, antwortet sie sofort. Die Ehefrau, der Vater oder die Tochter seien während der Therapie meistens anwesend. So habe sie die Gelegenheit, intensiv anzuleiten. Zum Beispiel, 


\section{Profession \& Perspektiven}

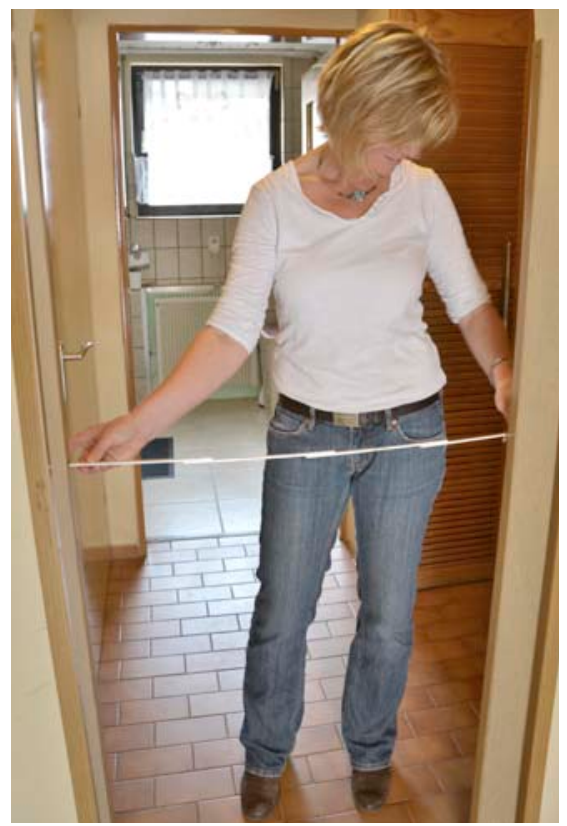

Die Therapeutin nimmt die häusliche Situation unter die Lupe. Reicht das für den Rollstuhl?

wie die Angehörigen den Transfer bewerkstelligen oder den Patienten in den Alltag einbinden können. Außerdem lege das Therapeutenteam viel Wert auf Aufklärungsarbeit. „Häufig erleben wir pflegende Angehörige am Rand ihrer Belastungsgrenze. Wir verwenden viel Zeit darauf, sie zu unterstützen und zu beraten“, erklärt die Ergotherapeutin. Hin und wieder berät sie die Angehörigen zusammen mit dem Kollegen vom Sozialdienst, organisiert einen Schnuppertag in der Tagespflege oder eine Nachbarschaftshilfe, um sie zu entlasten. Das bespricht sie frühzeitig im Team, denn die Organisation braucht Anlaufzeit. Oft auch Überzeugungsarbeit. Denn für viele - ob Angehörige oder Patienten - ist der Schritt in eine Tageseinrichtung eine Überwindung.

Neben der intensiven Arbeit mit den Angehörigen schätzt Katrin Storbeck vor allem die interdisziplinäre Zusammenarbeit. Sie sei viel enger als in der Klinik. „Und ich schaue viel mehr über meinen Tellerrand“, sagt sie. „Wir sind im ständigen Austausch, und jeder blickt hin und wieder über seinen typischen Fachbereich, um das zu erarbeiten, was die Patienten gerade brauchen." Wenn erforderlich, behandelt sie gemeinsam mit der Physio- therapeutin. Auf diese Weise können sie beispielsweise besser üben, mit Patienten Treppen zu steigen oder ins Auto ein- und auszusteigen. Der Erfolg gibt ihnen recht.

Die kollegiale Zusammenarbeit ist so intensiv, weil das Team recht klein ist. Das heißt, Katrin Storbeck hat immer dieselben Ansprechpartner und muss nicht wie in der Klinik erst herausfinden, welche Therapeuten zuständig sind. Man könnte meinen, eine mobile Reha wäre das Optimum. Doch da ist Katrin Storbeck anderer Meinung: „Generell ist so eine mobile Reha nicht für jeden angezeigt“, sagt sie. „Für viele ist die stationäre Reha genau richtig, insbesondere, wenn spezielle Therapiegeräte erforderlich sind. Unsere Patienten in der Mobilen Geriatrischen Rehabilitation sind einfach ein bisschen besonders und profitieren deshalb sehr davon.“

\section{Konkrete Unterstützung im Alltag > Nach} der Besprechung begleite ich Ergotherapeutin Silvia Hark aus dem Nord-Team auf einen Hausbesuch zu Ehepaar Schmid*, beide über 80 Jahre alt. Frau Schmid wurde nach einem Oberschenkelhalsbruch stationär behandelt und kam anschließend in die Kurzzeitpflege. Dort machte sie jedoch nur wenige Fortschritte, sodass das Team von der Mobilen Geriatrischen Reha sich dafür einsetzte, dass Frau Schmid nach Hause zu ihrem Mann kam und dort Therapie erhielt. Damit sie besser versorgt werden kann, steht das Pflegebett im Wohnzimmer. Hier übt Sylvia Hark mit ihr das Aufstehen. Den Ehemann leitet sie an, denn sein Einsatz ist gefragt, wenn seine Frau zur Toilette muss. Er ist ganz eifrig bei der Sache und unterstützt seine Frau, wo er nur kann. Viele Tipps der Ergotherapeutin haben die Schmids schon angenommen, zum Beispiel bei der Wohnraumanpassung, als es vor allem darum ging, die Wege zwischen Wohnzimmer, Bad und Küche so hindernisfrei wie möglich zu gestalten.

Sylvia Hark ist den ganzen Tag außer Haus und fährt ihre Route ab. „Wenn etwas Wichtiges wäre, kann ich jederzeit mit Christian Aldus telefonieren“, erzählt sie. Auch sie empfindet die Teamarbeit als sehr eng und wertvoll. Besonders gefällt ihr, dass es keine Konkurrenz zwischen den Berufsgruppen gibt, sondern viele

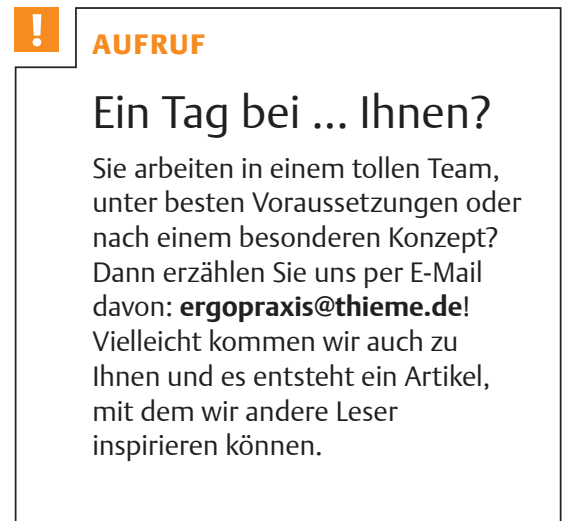

bereichernde Schnittstellen. Sie weiß aber auch, dass ihre Arbeitsbedingungen ganz besondere sind: „Diese Kommunikation ist in einer Praxis zeitlich nicht machbar, dafür braucht es ein eingespieltes Team vor Ort.“

Rehaklinik auf Rädern > Zurück im Hardtwaldzentrum treffe ich abschließend Ulrike Sinner, die Geschäftsführerin der Mobilen Ambulanten Rehabilitation Karlsruhe (MARK) und der Paritätischen Sozialdienste Karlsruhe. Sie ist stolz auf das multiprofessionelle Team unter der ärztlichen Leitung des Geriaters Christian Aldus: „Auf diese Weise können wir Energien bündeln und den Patienten einen enormen organisatorischen Aufwand abnehmen." Für sie ist die Mobile Geriatrische Reha eine Art „Rehaklinik auf Rädern“, die im Alltag der Menschen ansetzt und mit den unterschiedlichsten Kompetenzen auf deren Bedürfnisse eingeht. Und dass dieses Angebot gut ankommt, bestätigen die Rückmeldungen von Patienten und Angehörigen regelmäßig am Ende der Behandlung: „Können Sie nicht weitermachen?“, fragen sie dann. Ein Angebot, das Schule machen sollte. Wir brauchen definitiv mehr davon!

Simone Gritsch

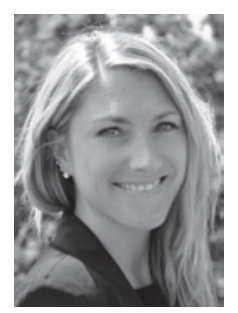

Simone Gritsch, Ergotherapeutin BcOT, hat die interdisziplinäre Zusammenarbeit in der Praxis immer vermisst. Dass das mit dem richtigen Konzept möglich ist, zeigte ihr der Besuch in Karlsruhe. 